\title{
PROGRAMA SAÚdE NA ESCOLA: ANÁLISE DA GESTÃO DAS AÇÕES DE PROMOÇÃO DA SAÚDE NO BRASIL
}

\section{SCHOOL HEALTH PROGRAM: ANALYSIS OF THE MANAGEMENT OF HEALTH PROMOTION ACTIONS IN BRAZIL}

\author{
Michely Karoline Ferraz Souto \\ Nutricionista Especialista em Formação Pedagógica \\ para Profissionais de Saúde, Faculdade Presidente Antônio Carlos \\ UNIPAC de Teófilo Otoni, Brasil. \\ E-mails: mifanikta@hotmail.com
}

Pâmela Francisco da Rocha

Nutricionista Especialista em Formação Pedagógica para Profissionais de Saúde, Faculdade Presidente Antônio Carlos

UNIPAC de Teófilo Otoni, Brasil.

E-mails: pampamfrocha@hotmail.com

\section{Karine Rodrigues da Silva Neumann}

Nutricionista Especialista em Vigilância em Saúde, Faculdade Presidente Antônio Carlos UNIPAC de Teófilo Otoni, Brasil. E-mails: krsnut@yahoo.com.br Recebido: 00/00/0000 - Aceito: 00/00/0000

\section{Resumo}

O presente artigo é produto de um estudo no entorno da gestão da Promoção da Saúde no Programa de Saúde na Escola (PSE), que estabelece uma articulação política intersetorial entre os Ministérios da Saúde e da Educação, tendo como eixo estruturante de suas ações a integração dos setores saúde e educação, através das Equipes de Saúde da Família e da Educação. Para o desenvolvimento da pesquisa, procurou explicar o problema a partir de uma pesquisa bibliográfica. $\mathrm{O}$ objetivo do presente estudo é analisar como é feita a gestão das ações de saúde propostas no 
Programa Saúde na Escola através da perspectiva da Promoção da Saúde, identificando as dificuldades do Grupo de Trabalho Intersetorial para realização das ações, analisando o envolvimento dos atores responsáveis pelo planejamento e operacionalização das ações de PS no PSE e as dificuldades vivenciadas pelos profissionais executantes das ações do PSE. Por meio deste estudo foi possível perceber que a Promoção da Saúde é uma importante ferramenta de enfrentamento, pois propõe ações que envolvem os aspectos da prevenção, promoção e atenção, mas infelizmente o programa esbarra em muitas dificuldades, não é fácil administrar uma política com articulação intersetorial onde o número de profissionais disponíveis não é suficiente para execução das propostas, as agendas dos setores são previamente montadas, tudo isso dificulta o envolvimento e comprometimento setorial e impede a sustentabilidade das ações.

Palavras-chave: Promoção da Saúde; Programa Saúde na Escola; Intersetorialidade

\section{Abstract}

This article is the product of a study surrounding the management of Health Promotion in the School Health Program (PSE), which establishes an intersectoral political articulation between the Ministries of Health and Education, with the integration of their actions as a structuring axis. health and education sectors, through the Family Health and Education Teams. For the development of the research, he tried to explain the problem from a bibliographic research. The objective of the present study is to analyze how the health actions proposed in the Health at School Program are managed through the Health Promotion perspective, identifying the difficulties of the Intersectoral Working Group to carry out the actions, analyzing the involvement of the actors responsible for the planning and operationalization of the PS actions in the PSE and the difficulties experienced by the professionals performing the PSE actions. Through this study it was possible to realize that Health Promotion is an important coping tool, as it proposes actions that involve the aspects of prevention, promotion and care, but unfortunately the program comes up against many difficulties, it is not easy to manage a policy with articulation intersectoral where the number of available professionals is not enough to execute the proposals, the agendas of the sectors are previously set up, all of which hinders sectoral involvement and commitment and prevents the sustainability of actions.

Keywords: Health promotion; Health at School Program; Intersectoriality

\section{Introdução}

A proposta de desenvolvimento do presente estudo surgiu a partir da vivência na prática profissional, na área da saúde e educação, no qual pude perceber as dificuldades de implementar ações intersetoriais em ambos os setores, principalmente 
quando se trata do desenvolvimento de ações de Promoção da Saúde (PS) voltadas para o âmbito escolar.

A Promoção da Saúde é uma das estratégias para a melhoria da qualidade de vida das pessoas, mas para que isso aconteça, é necessário parcerias intersetoriais.

O Programa Saúde na Escola (PSE) é um exemplo de política intersetorial, foi criado em 2007 pelos Ministérios da Saúde e da Educação com objetivo de contribuir na atenção integral (prevenção, promoção e atenção) à saúde de estudantes do ensino público básico. Por isso, é de suma importância o comprometimento de todos os atores envolvidos para que aconteça um fortalecimento e reconhecimento das estratégias de corresponsabilização e territorialização no campo da saúde e educação, para a sustentabilidade do programa.

Logo, o objetivo do presente estudo é analisar como é feita a gestão das ações de saúde propostas no Programa Saúde na Escola através da perspectiva da Promoção da Saúde, identificando as dificuldades do Grupo de Trabalho Intersetorial para realização das ações, analisando o envolvimento dos atores responsáveis pelo planejamento e operacionalização das ações de PS no PSE e as dificuldades vivenciadas pelos profissionais executantes das ações do PSE.

Com essa pesquisa espera-se contribuir para a reflexão sobre a importância da articulação a partir da intersetorialidade no PSE entre os setores da Saúde e Educação.

\section{Promoção da Saúde}

A promoção da saúde já vem sendo estudada por alguns autores há um tempo. Dentre as políticas de promoção da saúde, está o Programa Saúde na escola, para que esse Programa tenha êxito, é necessária uma gestão intersetorial com abordagens intersetoriais.

Jamais se falou tanto sobre Promoção da Saúde (PS) como na década atual. Isso pelo fato que, promover saúde é uma estratégia complexa que exige o entendimento da relação do indivíduo ou das populações com a sua história, suas relações com o mundo, com necessidades, seus padrões de desenvolvimento, com seu ambiente sociocultural, direitos e condições de vida (MOURA, 2014).

A promoção da saúde vem sendo entendida nos últimos anos como uma estratégia relevante para enfrentar os vários problemas de saúde que afetam as pessoas. As definições atuais de promoção de saúde partem de uma concepção 
ampla do processo saúde-doença e de seus determinantes e condicionantes (BUSS, 2000).

Um dos eixos que a promoção da saúde carrega é fortalecer a ideia de autonomia dos indivíduos e dos grupos sociais, e ao mesmo tempo, perspectivas progressistas, evidenciando a elaboração de políticas públicas intersetoriais, voltadas para melhoria da qualidade de vida das pessoas (COUTO, 2016).

De acordo com Santos (2017), a Promoção de Saúde deve ser uma meta intersetorial, com a participação ativa da comunidade. Além disto, deve estabelecer o fortalecimento da ação comunitária, de políticas públicas saudáveis, desenvolvimento de habilidades pessoais e reorientação de serviços sanitários e sistemas de saúde.

Segundo Medeiros (2015), a promoção da saúde converge para políticas públicas saudáveis e espaços favoráveis que promovam o bem-estar e a qualidade de vida das pessoas considerando o meio que estão inseridos e convivem.

Promover saúde é aceitar o grande desafio de estimular um amplo processo que envolve atuações intersetoriais, articulação de parcerias e participação da população, objetivando responder mais efetiva e integralmente às necessidades dos indivíduos (PINTO et al., 2012).

A promoção de saúde hoje é vista como responsabilidade de muitos setores sociais, visto que o conceito ampliado de saúde a coloca como resultado de diversos fatores. Para que a Promoção de Saúde seja efetiva, é necessário a organização intersetorial do processo saúde-doença com ações desenvolvidas de forma conjunta entre as esferas de governo e outros órgãos (BRASIL, 2004).

A execução da mesma necessita da articulação do conjunto das políticas públicas e dos diferentes setores da sociedade visando à melhoria da qualidade de vida (MALTA et al., 2014). Neste sentido, apresenta evidencias claras de que esta é diretamente dependente de muitas necessidades e por isso requer uma ação intersetorial (SOUSA, 2014).

\subsection{A promoção da Saúde no âmbito escolar}

Historicamente, o ambiente escolar vem sendo utilizado para importantes práticas de saúde, por se tratar de um espaço onde os educandos convivem durante vários anos. (COUTO, 2016).

Ao passar dos tempos, programas de saúde escolar foram sendo elaborados, a partir das novas ideias e entendimento sobre a temática Promoção da Saúde (ILHA 
et al., 2014). Para o autor, por mais que o entendimento de saúde tenha sido ampliado, as ações mais relevantes são as ações curativas e assistenciais e as ações de promoção têm sido confundidas com as de prevenção.

Pode-se considerar que foi com a implantação do conceito de Escola Promotora de Saúde, desde a criação do projeto Promoção da Saúde do Ministério da Saúde, que as práticas pedagógicas passaram integrar o tema de educação em saúde, então como os Ministérios da Educação e da Saúde passaram a desenvolver vínculos de apoio (TUSSET, 2012).

Há muito tempo 0 ambiente escolar vem sendo utilizado para 0 desenvolvimento de práticas de saúde, porém tais práticas adotadas tiveram suas bases apoiadas no padrão tradicional de saúde escolar (CAVALCANTI; LUCENA; LUCENA, 2015).

Para Vieira (2013), a saúde não é responsabilidade exclusiva do setor saúde, e vai além de um estilo de vida saudável, na direção de um bem-estar global. Deste modo, a promoção da saúde passa a ser compreendida como o processo de capacitação da comunidade para atuar na melhoria da sua qualidade de vida e saúde, incluindo uma maior participação no controle deste processo.

Com o passar dos anos com a mudança da concepção de saúde, a visão do processo de ensino-aprendizagem também vem sendo modificada. Isso proporciona o desenvolvimento de uma nova visão para se pensar em saúde e educação, levando em conta suas conexões (GOMES, 2009).

Assim como a saúde requer a articulação coordenada de diferentes políticas sociais para o alcance da integralidade, o mesmo acontece com a educação. Educar não se limita à memorização de letras e regras, nem decorar fórmulas matemáticas e datas. Educação envolve conquistar liberdade de articular ideias, de pensar e de analisar a realidade viva (VIEIRA, 2013).

A eficácia da promoção da saúde é um desafio metodológico e político que tem a capacidade de proporcionar e gerar conhecimento de programas adaptados à realidade de cada âmbito, tendo como objetivo à implantação de métodos apropriados (AFONSO; TAVARES; LUIZA, 2013).

Dada a sua capacidade e abrangência, o setor de educação, é um parceiro muito importante para a realização das ações de promoção da saúde voltadas para fortalecimento das capacidades dos indivíduos, para a tomada de decisões favoráveis tanto para sua saúde quanto para a comunidade, para a criação de espaços saudáveis e também para a consolidação de uma política intersetorial voltada para a qualidade 
De modo geral, todos os modelos de saúde na escola pressupõem uma proposta de articulação intersetorial entre Educação e Saúde. Entretanto, em sua maioria, isto não se traduz numa prática intersetorial inovadora e efetiva. (SILVA, 2010).

De acordo com Santos (2017), a promoção de saúde está sendo construída e aceita por outras áreas do conhecimento, haja vista que o setor saúde sozinho não é capaz de responder por todos os problemas de saúde. Portanto, destaca-se a importância da intersetorialidade, proposta desde a carta de Ottawa, nela o papel da escola como aliada e como sendo um cenário estratégico para prática de promoção de saúde.

No contexto brasileiro as políticas de saúde têm se desenvolvido no reconhecimento da intersetorialidade. Nunca se falou tanto em saúde e promoção da saúde como no contexto atual, o que comprova a valorização do papel de promoção da saúde associada ao ambiente escolar como elemento transformador da realidade. Dessa forma, reconhece-se a necessidade de atuação não somente do setor saúde, mas também como o resultado de ações intersetoriais

e multidisciplinares, tornando a intersetorialidade uma condição para a prática da Promoção da Saúde (FARIAS, 2016).

Para que a promoção da saúde seja concretizada é preciso articulação de diversos setores, bem como pessoas para realização de ações intersetoriais com vistas para alcançar os objetivos que a política de promoção da saúde propõe. Assim, o ambiente escolar aparece como um importante cenário para a promoção da saúde (CAVALCANTI; LUCENA; LUCENA, 2015).

\section{Programa Saúde na Escola}

O Ministério da Saúde e o Ministério da Educação, com propósito de ampliar as ações de saúde aos alunos da rede pública de ensino, criaram o Programa Saúde na Escola (PSE), com objetivo da formação integral dos estudantes da Educação Básica por meio de ações de prevenção, promoção e atenção à saúde (OLIVEIRA, 2017). O PSE foi estabelecido pelo decreto presidencial № 6.286 , de 5 de dezembro de 2007 (BRASIL, 2008), com o objetivo de estabelecer a intersetorialidade entre a 
saúde e educação e colaborar com a formação dos estudantes da rede pública (ANDRADE, 2004).

De acordo com Vieira (2013), o PSE foi idealizado para fortalecer a integração de duas políticas públicas, a saúde e a educação.

Em relação ao planejamento das ações, o Decreto Presidencial destaca que é preciso considerar o contexto escolar e social, o diagnóstico de saúde, e a capacidade operativa em saúde dos estudantes, bem como a inclusão das temáticas de educação em saúde no projeto político pedagógico das escolas. Além disso, as ações do Programa devem levar em consideração, também, o respeito à competência política dos Estados e municípios, à diversidade sociocultural das diferentes regiões do país e à autonomia dos educadores e das equipes pedagógicas (BRASIL, 2011).

Segundo Souza (2012), a operacionalização das ações desse programa acontece a partir da integração das Unidades Básicas de Saúde com as escolas. Essas ações devem ser desenvolvidas na escola de maneira mais articulada possível, aproximando todos os envolvidos.

As estratégias gerais de operacionalização das ações de promoção da saúde do escolar, descritas pelo Ministério da Saúde, define como ponto de partida a elaboração de um projeto que objetive a estruturação das ações a serem realizadas dentro do PSE (BRASIL, 2009).

O PSE deve ser executado em concordância com os princípios e normas do SUS e com a participação legítima das Equipes de Saúde da Família (ESF), de acordo com modelo de saúde vigorante que necessitarão desenvolver as ações de saúde propostas através do PSE, nos territórios estabelecidos de acordo com a área de domínio das Equipes de Saúde da Família, tornando possível a criação de associação entre os equipamentos públicos da saúde e da educação (FIGUEIREDO; MACHADO; ABREU, 2010).

O município interessado em aderir ao Programa Saúde na Escola, deve organizar um Grupo de Trabalho Intersetorial (GTI), que será encarregado pela gestão e por arquitetar um projeto que apresente o diagnóstico situacional das escolas locais (BRASIL, 2009). Santos (2017) ressalta, que a proposta de gestão do PSE por meio dos GTI é fundamentada em uma gestão compartilhada, que presume a realização coletiva do planejamento e execução das ações, de forma a atender às necessidades e demandas da região.

O GTI é composto obrigatoriamente por representantes das Secretarias de Saúde e de Educação e, facultativamente, por outros parceiros locais representantes 
somente mediante a assinatura do termo de compromisso pelos estados, Distrito Federal e municípios (BRASIL, 2011).

Com o objetivo de enfrentar as vulnerabilidades que possam prejudicar o desenvolvimento das crianças, adolescentes e jovens, o PSE preconiza o desenvolvimento integral dos escolares, juntamente a escola, elaborando programas e projetos, articulando saúde e educação. As ações que já estão em funcionamento entre saúde e a educação no ambiente escolar que possam ter impacto positivo, o PSE deverá acolher (BRASIL, 2019). As equipes de saúde de atenção básica e os profissionais de educação no território de educação constituem uma importante estratégia do PSE, com objetivo de assegurar a sustentabilidade das ações, a partir da responsabilidade sobre o território (BRASIL, 2011).

\subsection{Gestão do Programa Saúde na Escola}

De acordo com Neto (2015), o PSE utiliza o modelo de gestão compartilhada nas esferas municipal, estadual e federal, cada uma com sua importância para 0 efetivo sucesso do programa. Em cada esfera existe um Grupo de Trabalho Intersetorial (GTI), que é formado por no mínimo um representante do ministério da saúde, do ministério da educação, pais e outros. Onde no trabalho e no diálogo em conjunto terão possibilidade de aprofundar conhecimentos e estabelecer práticas para tratar as vulnerabilidades de cada região.

Na gestão do trabalho dos GTI é importante que o planejamento e as ações dentro do trabalho de gestão dos GTI sejam pautados nos componentes balizadores do PSE, sendo eles: a avaliação das condições de saúde, a prevenção de doenças e agravos e promoção de saúde, bem como a formação e capacitação dos profissionais que atuam ou atuaram no programa (NETO, 2015).

Os gestores podem ser considerados como instrumentos fundamentais para coordenar ações intersetorias, já que em nível local é possível reunir atores sociais em volta de problemas de sua realidade e buscar resultados próprios, direcionando o esforço em grupo para as ações que influenciam na qualidade de vida da população (FARIAS et al., 2016).

A gestão fará toda a diferença na condução no PSE, uma vez que não seja seguido às responsabilidades de qualquer que seja a esfera, irá refletir no resultado posterior, comprometendo dessa forma, todas as etapas. 


\section{Intersetorialidade}

As ações intersetoriais, são utilizadas como estratégias para atingir a saúde, e pode ser definida como um processo em que os objetivos e os recursos de cada unidade se consideram conforme suas repercussões e resultados nos objetivos, atividades e recursos dos outros setores (SANTOS, 2017).

A intersetorialidade é fundamentada em uma prática integrada de ações de diferentes setores, que se articulam, interagem e complementam para uma aproximação no enfretamento dos problemas, compartilhando metas e recursos, e desenvolvendo estratégias de forma conjunta (FARIAS et al., 2016).

De acordo com Vieira (2013), a intersetorialidade associa a ideia de integração, de equidade, de território e de direitos sociais. Isso faz com que os sujeitos que exercem as políticas públicas estejam de fato dispostas e determinadas a construir e articular experiências e saberes em todos os níveis do processo, desde o planejamento até a avaliação das ações. Atuar intersetorialidade requer mudanças nas práticas e na forma das organizações gestoras de políticas públicas.

No campo da Promoção da Saúde, as práticas intersetorias, têm como objetivo proporcionar a gestão partilhada entre usuários, movimentos sociais, trabalhadores do setor sanitário e de outros setores, produzindo autonomia e coparticipação. Entretanto, não é tarefa fácil superar o modelo setorial para o intersetorial, pois necessita da aceitação das partes envolvidas (TUSSET, 2012).

A interação entre os profissionais da saúde e da educação visa possibilitar entendimentos, habilidades complementares e pontos de vistas, com a intenção de gerar alternativas criativas em busca de saúde e educação integrais dos alunos (BRASIL, 2011).

A literatura mostra que o excesso de atividades no serviço e a sobrecarga na agenda é um motivo importante que atrapalha a articulação de vários trabalhos que exigem intersetorialidade, principalmente em ações intersetoriais envolvendo saúde e educação, áreas que tradicionalmente são sobrecarregadas de funções, com poucos recursos humanos e rotinas diferentes (TUSSET, 2012).

Teixeira (2004) destaca que, as práticas intersetoriais, implicam um impacto expressivo quando as ações priorizadas são estimuladas e tem por finalidade à solidificação da integralidade da atenção à saúde. Deste modo, as necessidades, 
demandas sociais dos diversos grupos da população e reorganização das respostas aos problemas, são representados pela intersetorialidade.

Diversos estudos apontam para a necessidade de que a intersetorialidade seja utilizada como instrumento de gestão, ao invés de ser reduzida a uma mera responsabilidade dos profissionais. Para a adoção da intersetorialidade na gestão pública é necessário priorizar algumas ações e práticas, são elas: mudança de paradigma, ou seja, criação de uma nova forma de pensar, que leve em consideração a complexidade das demandas sociais; projeto político transformador com o intuito de dar impulso à questão da inclusão social e da equidade, superando a existência das políticas assistencialistas; planejamento e avaliação participativos e com base regional, com vistas a atender às necessidades locais; e a atuação em rede de compromisso social, no intuito de favorecer a mobilização dos atores sociais ligados ao aparato governamental e social, para que juntos possam trabalhar as problemáticas da sociedade (CAVALCANTI; LUCENA, 2015, p.394).

A intersetorialidade é uma condição para o desenvolvimento de programas como o Programa Saúde na Escola, que tem na PS um de seus horizontes. Para que aconteça o desenvolvimento de ações intersetoriais, é de suma importância que todos os setores envolvidos estejam, de fato, compartilhando suas metas, seus objetivos, os processos, as atividades, as dúvidas, os resultados, os erros e os acertos (SILVA et al., 2013).

\section{Resultados e discussão}

Este estudo buscou colaborar com a discussão sobre intersetorialidade na gestão e a sua operacionalização nas práticas de Promoção da Saúde voltadas para a escola.

A articulação intersetorial entre atenção básica e rede escolar, constitui-se em um eixo estratégico para a construção de ações de PS no âmbito escolar.

Apesar dos avanços que constam nos registros referentes ao desenvolvimento dos programas voltados para a saúde escolar, nota-se que estes ainda contam com falhas e deficiências, e muitas vezes não se adequam às prioridades e oportunidades 
planos de estudo que sejam convergentes com as necessidades que apresentam (CAVALCANTI; LUCENA; LUCENA, 2015).

Para Moura (2014), os profissionais de saúde e educação enfrentam rotineiramente em seu local de atuação muitos impedimentos que dificultam a execução de seus trabalhos com qualidade como: falta de recursos financeiros, estrutura física inadequada, materiais para procedimentos, baixa remuneração e recursos humanos.

Ao analisar fatores facilitadores e dificultadores do trabalho das equipes da ESF, Silva et al., (2013), encontra questionamentos em relação a falta de tempo para o desenvolvimento das várias tarefas executadas nas unidades de saúde.

Segundo Farias et al. (2016), o diálogo entre os setores de saúde e educação para a formulação, implantação, acompanhamento ou avaliação conjunta de políticas e programas de PS no cenário da escola ainda é um grande desafio para o PSE. A falta de tempo e a sobrecarga de trabalho podem ser entendidas como fatores coadjuvantes na ausência de envolvimento total. De acordo com o autor, pensar em estratégias para incrementar esse diálogo seria importante, o autor acredita ser um ponto-chave para facilitar o refletir-agir e fortalecer as ações intersetoriais.

De acordo com Silva e Rodrigues (2010), a intersetorialidade ainda se consiste como um objetivo a ser atingido. Reconhecem que em um território existem muitas possibilidades de articulação em rede, ainda assim, para que o serviço de saúde local possa de fato firmar parcerias intersetoriais, é fundamental ir além da negociação de tarefas e responsabilidades.

A intersetorialidade deve ser construída de forma flexível, para isso os setores devem identificar onde existe mais problemas e elaborar estratégias de intervenção que ultrapassem os programas já implementados setorialmente e envolvam que envolvam os vários setores (GOMES, 2009).

A ampliação de ações nesta área, fomentada pelo Programa Saúde na Escola, necessita estar ancorada em processos de planejamento conjuntos entre os setores, tendo como ponto de partida as particulares necessidades de saúde emergentes nos territórios. Além disso, ainda existe um distanciamento entre os dois setores.

Vale ressaltar que a intersetorialidade deve estar presente no Programa Saúde na Escola desde seu planejamento, na execução, no monitoramento e até na avaliação das ações. Nenhum dos setores envolvidos deve agir passivamente, e sim unir para desenvolver ações de promoção da saúde impactantes, sempre buscando 
Apesar de todo o planejamento do programa apoiado em objetivos, ações e componentes bem definidos, o PSE se mostra como um desafio no cenário brasileiro tanto para gestores quanto para profissionais, pela complexidade da proposta e, principalmente pelas amplas demandas desempenhadas nos setores de saúde e educação (MARINHO; MACHADO; BARRETO, 2015).

O diálogo entre os setores é a chave para que haja uma responsabilidade compartilhada no trabalho do PSE, com gestores e profissionais refletindo na perspectiva de um processo de trabalho também interdisciplinar, trazendo resultados positivos para o programa.

\section{Considerações Finais}

No presente artigo verificou-se que a proposta de trabalho intersetorial ainda é um desafio a ser superado no Programa Saúde na Escola.

Diante do exposto, cabe aos gestores do PSE, o entendimento de corresponsabilização pela identificação de problemas, análise e proposição de soluções concretas que contribuam para a melhoria das ações ligadas à promoção da saúde na escola, tendo como bases os pressupostos do campo da gestão ligados à eficácia e eficiência dos recursos já disponíveis em políticas públicas de ambos os setores.

Portanto, surge a necessidade de reorganização e redirecionamento de práticas nos setores e ainda na gestão, de forma que cada ator envolvido compreenda suas funções e responsabilidades, busque o diálogo setorial e intersetorial e planeje as ações não apenas considerando as metas, mas principalmente as necessidades dos estudantes.

\section{Referências}

AFONSO, Carmelinda Monteiro Costa.; TAVARES, Maria de Fátima Lobato.; LUIZA, Vera Lúcia. Escolas promotoras da saúde na América Latina: uma revisão do período 1996- 2009. Fortaleza, 2013. Disponível em:

<http://periodicos.unifor.br/RBPS/article/view/2642/pdf>. Acesso em: 21 ago. 2018. 
ANDRADE, Luiz Odorico Monteiro de et al. Escola de Formação em Saúde da Família Visconde de Sabóia: três anos construindo a tenda invertida e a educação permanente no SUS. SANARE - Revista de Políticas Públicas. Sobral, v. 5, n. 1, p. 33-39, jan./mar. 2004. Disponível em: <https://sanare.emnuvens.com.br/sanare/article/view/120/112>. Acesso em: 24 set. 2018.

BRASIL. Revista Brasileira Saúde da Família. Programa Saúde na Escola. Brasília: Ministério da Saúde, v. 20 n. 9, out./dez. 2008, n. 20, out./dez. 2008. Disponível em: $<$ http://dab.saude.gov.br/portaldab/biblioteca.php?conteudo=publicacoes/revista_saud e_familia20> Acesso em: 23 set. 2018.

. Ministério da Saúde. Departamento de Atenção Básica. Saúde na Escola. Brasília, 2009. Disponível em:

<http://189.28.128.100/dab/docs/publicacoes/cadernos_ab/abcad24.pdf >. Acesso em 23 ago. 2018.

. Ministério da Saúde. Departamento de Atenção Básica. Passo a Passo do PSE. Programa Saúde: tecendo caminhos da intersetorialidade. Brasília, 2011. Disponível em: <http://189.28128.100/dab/docs/legislacao/passo_a_passo_pse.pdf>. Acesso em 23 ago. 2018.

BUSS, Paulo Marchiori. Promoção da Saúde e qualidade de vida. Ciência \& Saúde Coletiva. Rio de Janeiro, v. 5, n. 1, p. 163-177, 2000. Disponível em:

http://dx.doi.org/10.1590/S1413-81232000000100014 >. Acesso em: 21 agos. 2018.

CAVALCANTI, Patrícia Barreto.; LUCENA, Carla Mousinho Ferreira.; LUCENA, Pablo Leonid Carneiro. Programa Saúde na Escola: interpelações sobre ações de educação e saúde no Brasil. Textos \& Contextos. Porto Alegre, v.14, n.2, ago/dez, 2015. Disponível em:

<http://revistaseletronicas.pucrs.br/ojs/index.php/fass/article/view/21728/13961>. Acesso em: 20 agos. 2018.

COUTO, Analie Nunes et al. O ambiente escolar e as ações de promoção da saúde.

CINERGIS - Revista do Departamento de Educação Física e Saúde e do Mestrado em Promoção da Saúde da Universidade de Santa Cruz do Sul - Unisc. Rio Grande do Sul, v. 17, n. 4, out/dez, 2016. Disponível em:

$<$ https://online.unisc.br/seer/index.php/cinergis/article/view/8150>. Acesso em: 21 ago. 2018.

FARIAS, Isabelle Carolline Verissimo de et al. Análise da intersetorialidade no programa saúde na escola. Revista Brasileira de Educação Médica. Rio de Janeiro, v. 40, n. 2, abr/jun, 2016. Disponível em: <http://dx.doi.org/10.1590/1981-

52712015v40n2e02642014>. Acesso em: 23 set. 2018. 
FIGUEIREDO, Túlio Alberto Martins.; MACHADO, Vera Lúcia Taqueti.; ABREU, Margaret Mirian Scherrer. A saúde na escola: um breve resgate histórico. Ciência \& Saúde Coletiva. Rio de Janeiro, v. 15, n. 2, Mar, 2010. Disponível em: <http://dx.doi.org/10.1590/S1413-81232010000200015>. Acesso em: 21 ago. 2018.

GOMES, Mauro de Lima. Política Nacional de Promoção da Saúde: potência de transformação ou política secundária? 2009. 88 f. Dissertação (Mestrado em Políticas Públicas e Formação Humana). Universidade do Estado do Rio de Janeiro, Rio de Janeiro, 2009. Disponível em:

<http://www.ppfh.com.br/wpcontent/uploads/2014/01/D_politicanacional.pdf> Acesso em: 20 ago. 2018.

ILHA, Phillip Vilanova et al. Intervenções no ambiente escolar utilizando a promoção da saúde como ferramenta para a melhoria do ensino. Revista Ensaio. Belo Horizonte, v. 15, n. 2, p. 35-53, Set-Dez, 2014. Disponível em:

<http://www.scielo.br/pdf/epec/v16n3/1983-2117-epec-16-03-00035.pdf >. Acesso em: 20 ago. 2018.

MALTA, Deborah Carvalho et al. A implementação das prioridades da Política Nacional de Promoção da Saúde, um balanço, 2006 a 2014. Ciência Saúde Coletiva. Rio de Janeiro. v. 19, n. 11, p. 4301-4312, Nov, 2014. Disponível em: $<$ http://www.scielo.br/scielo.php?script=sci_arttext\&pid=S141381232014001104301\&l $\mathrm{ng}=\mathrm{en} \& \mathrm{nrm}=\mathrm{iso}>$. Acesso em: 26 set. 2018.

MARINHO, Mirna Neyara Alexandre de Sá Barreto.; MACHADO, Maria de Fátima Antero Souza.; BARRETO, Francisco Myellyson Alexandre de Sá. Desafios e limites ao programa saúde na escola (PSE) no Cariri Cearense. Ceará, 2015. Disponível em: <http://www.convibra.com.br/ upload/paper/2015/76/2015_76_11458.pdf >. Acesso em: 24 set. 2018.

MEDEIROS, Livia Maria Rodrigues de Pontes. A gestão do programa saúde na escola no município de Natal/RN: um estudo de caso. 2015. 83 f. Dissertação (Mestrado em Saúde Coletiva) - Programa de Pós-Graduação em Saúde Coletiva. Universidade Federal do Rio Grande do Norte, Natal, 2015. Disponível em: <https://repositorio.ufrn.br/jspui/handle/123456789/20521>. Acesso em: 27 set. 2018.

MOURA, Samara Marques de. Programa Saúde na Escola: um instrumento da intersetorialidade na promoção da saúde. 2014. 113 f. Dissertação (Mestrado em Odontologia) - Universidade Federal de Goiás, Goiânia, 2014. Disponível em: <https://repositorio.bc.ufg.br/tede/handle/tede/6893>. Acesso em: 24 set. 2018. 
NETO, Severino Santino do Nascimento. Programa saúde na escola no contexto da política pública. Campina Grande, 2015. Disponível em:

<http://dspace.bc.uepb.edu.br/jspui/handle/123456789/13124>. Acesso em: 24 set. 2018.

OLIVEIRA, Fernanda Piana Santos Lima. Avaliação do programa saúde na escola com foco na integração entre unidade básica de saúde e escola de ensino fundamental: um estudo de caso em Belo Horizonte, Brasil. 2017. 224 f. Tese (Doutorado em Saúde Coletiva) - Faculdade de Odontologia, Universidade Federal de Minas Gerais, Belo Horizonte, 2017. Disponível em:

<http://www.bibliotecadigital.ufmg.br/dspace/handle/1843/BUOS-ATXKKG>. Acesso em: 21 ago. 2018.

PINTO, Bruna Knob et al. Promoção da saúde e intersetorialidade: um processo em construção. Revista Mineira de Enfermagem remE. Belo Horizonte, v. 16, n.4, p. 487-493, Out-Dez, 2012. Disponível em: <http://www.reme.org.br/artigo/detalhes/552>. Acesso em: 27 ago. 2018.

SILVA, Carlos dos Santos.; PANTOJA, Avamar Filgueira. Contribuições da avaliação na identificação de efetividade e da promoção da saúde na escola no município do Rio de Janeiro. Senac: A Revista da Educação Profissional. Rio de Janeiro, v. 35, n. 2, p.37-49 Mai-Ago, 2009. Disponível em:

<http://www.bts.senac.br/index.php/bts/article/view/245/241 >. Acesso em: 27 set. 2018.

SILVA, Kênia Lara.; RODRIGUES, Andreza Trevenzoli. Ações intersetoriais para promoção da saúde na Estratégia Saúde da Família: experiências, desafios e possibilidades. Revista Brasileira de Enfermagem. Brasília, v. 63, n. 5, p. 762-769, Set-Out, 2010. Disponível em: <http://www.scielo.br/pdf/reben/v63n5/11.pdf>. Acesso em: 25 set. 2018.

SILVA, Kênia Lara et al. Promoção da Saúde e Intersetorialidade em um Município da Região Metropolitana de Belo Horizonte/MG. Revista de APS. Belo Horizonte, v. 16, n. 2, p. 165-172, abr./jun. 2013. Disponível em:

$<$ https://aps.ufjf.emnuvens.com.br/aps/article/view/1757/717>. Acesso em: 27 set. 2018.

SANTOS, Lúcia de Fátima da Silva. As ações do Programa Saúde na Escola na perspectiva dos profissionais da saúde e da educação. 2017. 82 f. Dissertação (Mestrado em Saúde e Comunidade) - Universidade Federal do Piauí, Teresina, 2017. Disponível em: 
<http://repositorio.ufpi.br/xmlui/bitstream/handle/123456789/1011/As\%20a\%C3\%A7\%C 3\%B5es\%20do\%20Programa\%20Sa\%C3\%BAde\%20na\%20Escola\%20na\%20perspe ctiva\%20dos\%20profissionais\%20da\%20sa\%C3\%BAde\%20e\%20da\%20educa\%C3\% A7\%C3\%A3o.pdf? sequence=1>. Acesso em: 20 agos. 2018.

SOUSA, Marta Caires de. Saúde na Escola: analisando os caminhos da intersetorialidade. 2014. 100 f. Dissertação (Mestrado em Saúde Comunitária) Instituto de Saúde Coletiva, Universidade Federal da Bahia, Salvador, 2014. Disponível em:

<https://repositorio.ufba.br/ri/bitstream/ri/16334/1/DISSERTA\%C3\%87\%C3\%830\%20 MARTA\%20CAIRES.\%202014.pdf>. Acesso em: 21 set. 2018.

SOUZA, Nathália Paula de. Avaliação das ações de saúde e nutrição na perspectiva do programa saúde na escola. 2012. 127 f. Dissertação (Mestrado em Nutrição) - Universidade Federal de Pernambuco, Recife, 2012. Disponível em: <https://repositorio.ufpe.br/handle/123456789/11789>. Acesso em: 24 set. 2018.

TEIXEIRA, Carmen Fontes. Formulação e implementação de políticas públicas saudáveis: desafios para o planejamento e gestão das ações de promoção da saúde nas cidades. Saúde e Sociedade. São Paulo, v. 13 n. 1, p. 37-46, jan/abr. 2004. Disponível em: <http://www.scielo.br/pdf/sausoc/v13n1/05.pdf>. Acesso em: 23 agos. 2018.

TUSSET, Dalila. Competências em promoção da saúde no programa saúde na escola no Distrito Federal. 2012. 161 f. Dissertação (Mestrado em Educação Física) Universidade de Brasília, Distrito Federal, 2012.

Disponível em: <http://repositorio.unb.br/handle/10482/11930>. Acesso em: 20 agos. 2018.

VIEIRA, Maria Edna Moura. Programa Saúde na Escola: a intersetorialidade em movimento. 2013. 94 f. Dissertação (Mestrado em Processos de Desenvolvimento Humano e Saúde) - Universidade de Brasília, Brasília, 2013. 